\title{
Physico-chemical modification of kafirin microparticles and their ability to bind bone morphogenetic protein-2 (BMP-2), for application as a biomaterial
}

\author{
Joseph O. Anyango ${ }^{\dagger}$ Nicolaas Duneas, ${ }^{\ddagger}$ John R.N. Taylor, ${ }^{\dagger}$ Janet Taylor, ${ }^{\dagger} *$ \\ ${ }^{\dagger}$ Institute for Food, Nutrition and Well-being and Department of Food Science, University of Pretoria, Private Bag X20, \\ Hatfield 0028, South Africa. \\ ॠ Altis Biologics, 9 Herbert Baker St, Groenkloof, South Africa \\ *Corresponding author Phone: +27 12420 5402, Fax: +27 124202839 \\ E-mail: janet.taylor@up.ac.za (Janet Taylor)
}

\begin{abstract}
Vacuolated spherical kafirin microparticles mean diameter $5 \mu \mathrm{m}$, can be formed from an acidic solution with water addition. Three dimensional scaffolds for hard tissue repair require large structures with a high degree of interconnected porosity. Cross-linking the formed kafirin microparticles using wet heat or glutaraldehyde treatment resulted in larger structures (approx. 20 $\mu \mathrm{m})$, which whilst similar in size and external morphology, were apparently formed by further assisted-assembly by two significantly different mechanisms. Heat treatment, which increased vacuole size, involved kafirin polymerization by disulfide bonding with the microparticles being formed from round, coalesced nanostructures, as shown by AFM. Kafirin polymerization of glutaraldehyde treated microparticles was not by disulfide bonding and the nanostructures, as revealed by AFM, were spindle shaped. Both treatments enhanced BMP-2 binding to the microparticles, probably due to their increased size. Thus, these modified kafirin microparticles have potential as natural, non-animal protein bio-active scaffolds.
\end{abstract}

KEYWORDS: kafirin, microparticle, cross-linking, binding, BMP-2 


\section{INTRODUCTION}

Cereal prolamin proteins, such as zein and kafirin, can be formed into nano- and microparticles (1, 2). These particles have potential applications as delivery systems for drugs (1), nutraceuticals (3, 4), antimicrobials (5), essential oils (6), as biomaterials in tissue engineering as scaffolds (7), and as biomedical coatings for arterial/vascular prostheses (8). Zein and kafirin are natural, plant based, non-allergenic, slowly biodegradable and have some advantages over animal based biomaterials such as silk and collagen, especially for biomedical applications. Collagen and silk have poor wet strength and bovine collagen has potential immunogenicity and has been reported to transmit diseases such as bovine spongiform encephalopathy (as reviewed by 9).

Wang and Padua et al. (10) recently described a possible nano-scale mechanism for the selfassembly of various zein mesostructures. The self-assembly of these zein structures appears to be driven by the amphiphilic nature of the protein and occurred when changes were made to the polarity of an aqueous ethanol solution of zein by evaporation. An earlier paper by the same workers indicated that, at larger scale, zein microspheres self-assembled by layering onto a central core (11). They suggested radial growth occurred by hydrophobic interactions as the solvent became more hydrophilic due to the evaporation of the ethanol.

Kafirin is very similar to zein in amino acid composition but is more hydrophobic, or more strictly speaking less hydrophilic (12) and so can be considered amphiphilic in nature. Kafirin microparticles can be made by a process that is almost opposite to that used by Wang and Padua et al. $(10,11)$. Instead of dissolving the protein in aqueous ethanol and increasing the polarity of the solution by evaporation of the ethanol, the kafirin is dissolved in a primary solvent, glacial acetic 
acid, water is then added resulting in the formation of kafirin microparticles (2). In both cases there is a change in solvent polarity, causing microparticle formation as described by Wang and Padua $(10,11)$ for zein.

Comparison of the structure of zein and kafirin microparticles show that the kafirin microparticles are generally larger (1-10 microns), (2) than zein microparticles (ranges from 0.3-1.7 microns), (1) when made by similar processes. Kafirin microparticles have a rough surface and internal vacuoles resulting in a large surface area, whereas, zein microparticles are generally smooth and solid (6). The vacuoles in the kafirin microparticles are thought to be the foot print of air bubbles incorporated in the very viscous protein solution which become entrapped within the microparticles as they form (2).

Although there is considerable interest in nano-sized particles, some potential biomaterial applications, particularly for three dimensional scaffold type structures for hard tissue repair, require large particles with a high degree of interconnected porosity (13). An example would be an injectable dental implant, particle size range of $80 \mu \mathrm{m}$ to $200 \mu \mathrm{m}$ (14).

Cross-linking by physical (15), chemical (16) and enzymic (15) methods have been applied to water soluble protein nano and microparticles, such as whey protein(15) and gelatin (16), to increase water resistance and reduce swelling. However, there has been little research on cross-linking of prolamin protein microparticles, probably because the proteins are relatively hydrophobic (12). Various methods of cross-linking kafirin microparticles have been investigated to improve the water resistance of bioplastic films made from them (17). Cross-linking has also been investigated to improve the tensile properties of cast kafirin films (18) and cast zein films (19). Also, zein fibers 
have been cross-linked to improve their mechanical properties (20), water stability (21), and cytocompatibility (22).

The main objectives of this research were to determine whether cross-linking can increase the size of kafirin microparticles to improve their potential utility as biomaterial scaffolds and to attempt to understand the underlying mechanism involved.

Bone morphogenetic proteins (BMPs) induce the formation of both cartilage and bone and also play a role in a number of non-osteogenic developmental processes such as in neural induction (23). BMP-2 is a potent osteogenic growth factor that has been approved for clinical use (24). Mammalian collagen, the commonly used carrier for BMPs (24) may potentially induce immune response through cross-reactivity with human cells and also has a risk of disease transmission (25). Therefore, there is a need for delivery systems that are phylogenetically far from human tissue. Thus, an additional objective of this study was to determine whether kafirin microparticles could bind to BMP-2 and thus have the potential as a carrier scaffold for BMPs for tissue repair.

\section{MATERIALS AND METHODS}

Materials. Kafirin was extracted from a mixture of grain of two similar white, tan-plant non-tannin sorghum cultivars PANNAR PEX 202 and 606, as described (17). The protein was defatted and freeze-dried. The protein content $(\mathrm{N} \times 6.25$, dry matter basis $)$ determined by Dumas nitrogen combustion method (26).

Kafirin microparticles were prepared essentially according to Taylor et al. (2) with slight modification. Kafirin was dissolved in glacial acetic acid (32\% protein (w/w) solution) and 
equilibrated overnight at $22^{\circ} \mathrm{C}$. Distilled water was then added to the kafirin solution, at rate of 1.4 $\mathrm{mL} /$ min using a Watson-Marlow Bredel peristaltic pump (Falmouth, UK) and mixing using a magnetic stirrer at $600 \mathrm{rpm}$ to form the kafirin microparticle suspension. The suspension contained $2 \%(\mathrm{w} / \mathrm{w})$ kafirin protein in $0.9 \mathrm{M}$ acetic acid (final $\mathrm{pH} 2.0$ ). Freeze dried microparticles were prepared by removing the acid by centrifugation at $3150 \mathrm{~g}$ for $10 \mathrm{~min}$ and washing the pellet of microparticles three times with distilled water. The supernatant was removed before freeze drying. Kafirin microparticles were stored at $10^{\circ} \mathrm{C}$ before analysis.

Heat or glutaraldehyde treatment during microparticle preparation. Hot distilled water $\left(96^{\circ} \mathrm{C}\right)$ or a solution of $6.85 \%(\mathrm{w} / \mathrm{w})$ glutaraldehyde was added to the solution of kafirin in glacial acetic acid $(24 \% \mathrm{w} / \mathrm{w})$ using a peristaltic pump at the rate of $1.4 \mathrm{~mL} / \mathrm{h}$, while stirring, to give a final kafirin concentration of $2 \%(\mathrm{w} / \mathrm{w})$ in $0.9 \mathrm{M}$ acetic acid (final $\mathrm{pH} 2.0$ ). The final temperature of the heat treatment was approximately $75^{\circ} \mathrm{C}$ and the final glutaraldehyde concentration was $75 \%(\mathrm{w} / \mathrm{w})$ (protein basis). Lower glutaraldehyde concentrations did not increase kafirin microparticle size (data not shown).

Wet heat treatment of kafirin microparticles. A kafirin microparticle suspension was prepared and washed free of acetic acid, as described above. The resultant pellet was re-suspended in $91 \%$ $(\mathrm{w} / \mathrm{w})$ water $(\mathrm{pH}$ 6.74). Wet heat treatment was carried out by heating the kafirin microparticle suspensions at $50^{\circ} \mathrm{C}, 75^{\circ} \mathrm{C}$ and $96^{\circ} \mathrm{C}$ for $1 \mathrm{~h}$. A control sample was maintained at $22^{\circ} \mathrm{C}$.

Glutaraldehyde treatment of kafirin microparticles. Kafirin microparticle suspensions were prepared as described above. Glutaraldehyde was added to $4.0 \mathrm{~g}$ kafirin microparticle suspensions containing $2 \%$ protein in $(\mathrm{w} / \mathrm{w})$ acetic acid $(\mathrm{pH} 2.0)$, resulting in final glutaraldehyde concentrations 
of $0 \%, 10 \%, 20 \%$, and $30 \%(\mathrm{w} / \mathrm{w})$ on protein basis. Samples were then vortex-mixed and held at $22^{\circ} \mathrm{C}$ for $12 \mathrm{~h}$.

SDS-PAGE. Treated kafirin microparticles were characterized by SDS-PAGE (16) under reducing and non-reducing conditions on preprepared 4-12\% Bis-Tris gradient gels (Invitrogen Life Technologies, Carlsbad, CA) using an X Cell SureLock Mini-Cell electrophoresis unit (Invitrogen Life Technologies). The loading was $\approx 10 \mu \mathrm{g}$ protein. Invitrogen Mark12 ${ }^{\mathrm{TM}}$ Unstained Standard was used. Proteins were stained with Coomassie ${ }^{\circledR}$ Brilliant Blue R250 and scanned on a flat-bed scanner.

Scanning Electron Microscopy (SEM) and Transmission Electron Microscopy (TEM). Suspensions of kafirin microparticles were prepared for SEM and TEM according Taylor et al. (2) SEM preparations were viewed using a Jeol JSM-840 Scanning Electron Microscope (Tokyo, Japan). TEM preparations were viewed with a Jeol JEM-2100F Field Emission Electron Microscope (Tokyo, Japan).

Atomic force microscopy (AFM). Freeze-dried kafirin microparticles were embedded on the surface of aluminum stubs using double-sided tape. Then the microparticles were viewed with a Veeco Icon Dimension Atomic Force Microscope (Bruker, Cambridge, UK) using tapping in air mode. A $8 \mathrm{~nm}$ silicon tip on nitride lever cantilever was used.

Microparticle size was determined by comparing their images with that of a scale bar of the same magnification. At least 100 microparticles were measured of duplicate samples of each treatment. In vitro protein digestibility (IVPD) was performed on freeze-dried kafirin microparticles using a microscale pepsin digestion protocol (27) modified from Hamaker et al. (28). P7000-100G pepsin (Sigma, Johannesburg, South Africa), activity 863 units/mg protein, was used. 
Fourier transform infrared (FTIR) spectroscopy was performed as described by Taylor et al. (2). Freeze-dried microparticles were scanned using a Vertex 70v FT-IR spectrometer (Bruker Optik, Ettlingen, Germany), using 64 scans, $8 \mathrm{~cm}^{-1}$ band and an interval of $1 \mathrm{~cm}$ in the Attenuated Total Reflectance (ATR) mode. The FTIR spectra were Fourier-deconvoluted with a resolution enhancement factor of 2 and $12 \mathrm{~cm}^{-1}$ bandwidth.

Kafirin microparticles as a potential delivery system for BMP-2. Control, heat $\left(75^{\circ} \mathrm{C}\right)$ and glutaraldehyde (20\%) treated kafirin microparticles were used for the BMP binding study. The carrier medium for kafirin microparticles was exchanged from $0.9 \mathrm{M}$ acetic acid to $20 \mathrm{mM}$ acetic acid by centrifuging the microparticle suspension at $3150 \mathrm{~g}$ for $20 \mathrm{~min}$, decanting off the supernatants and re-suspending the microparticles in $20 \mathrm{mM}$ acetic acid, re-centrifuging and then replacing the supernatant with fresh $20 \mathrm{mM}$ acetic acid. This process was repeated three times. The volume of $20 \mathrm{mM}$ acetic acid was adjusted to give a solid content of at least $6 \%(\mathrm{w} / \mathrm{w})$.

Binding BMP-2 with kafirin microparticles. Kafirin microparticle suspensions containing 100 mg protein or $100 \mathrm{mg}$ porcine collagen standard (Altis Biologics, Pretoria, South Africa) were weighed into $5 \mathrm{~mL}$ cryovials, $0.5 \mathrm{~mL} 1000$ ppm Tween ${ }^{\circledR} 20$ was added and vortex-mixed. Porcine BMP complex (Altis Biologics) $(1.5 \mathrm{~mL})$ was added to kafirin microparticles or collagen standard and vortex-mixed to give a BMP complex to carrier protein ratio of $\approx 1: 100$ in $4.5 \mathrm{~mL} 20 \mathrm{mM}$ acetic acid. An initial $50 \mu \mathrm{L}$ sample was drawn from unbound sample and transferred into microwells of a microplate (Greiner Bio-One, Frickenhausen, Germany) to account for time 0 from each treatment. The cryovials were placed on a rocking platform (50 rpm). After 30, 120 and 1440 min, samples were centrifuged at $3150 \mathrm{~g}$ for $20 \mathrm{~min}$ and $50 \mu \mathrm{L}$ sample material drawn from clear supernatants of each sample treatment and transferred into microwells of a microplate. For confirmation of binding BMP-2 with kafirin microparticles, enzyme-linked immunosorbent assay (ELISA) was used to 
measure the amount of BMP-2 in the supernatants of the various treatments. A Quantikine ${ }^{\circledR}$ BMP-2 Immunoassay kit (R\&D Systems, Minneapolis, MN) was used.

Statistical analyses. One-way analysis of variance (ANOVA) using Fischer's Least Significant Difference (LSD) test was used to analyze data with Statistica version 10 software (StatSoft, Tulsa, OK). Experiments were carried out in duplicate.

\section{RESULTS AND DISCUSSION}

Heat and glutaraldehyde treatments during microparticle preparation. Kafirin microparticles prepared at ambient temperature $\left(22^{\circ} \mathrm{C}\right)$ were spherical, between 1 and $10 \mu \mathrm{m}$ in diameter, with pores (vacuoles) between 0.5 and $2 \mu \mathrm{m}$ (Figure 1A, B ), as described previously Taylor et al. (2). The majority of the microparticles were between 1 and $5 \mu \mathrm{m}$. The heat- and glutaraldehyde-formed particles were only slightly larger, with the glutaraldehyde-formed microparticles being fairly uniform in size (Figure 1E, F), whereas-the heat formed microparticles were less homogeneous (Figure 1C, D). In terms of appearance, the heat- and glutaraldehyde-formed microparticles differed from the untreated control in that they had smooth surfaces and fewer internal vacuoles. Since the aim of the study was to substantially increase the size of the microparticles, the approach of crosslinking after microparticle formation was investigated.

Heat Treatment following microparticle preparation. Wet heat treatment following the microparticle formation changed the shape of the larger kafirin microparticles to oval and increased their average size to $20 \mu \mathrm{m}$ (Figure $2 \mathbf{C}, \mathbf{F}$ ). Particle size increased with increasing severity of the heat treatment up to $75^{\circ} \mathrm{C}$ (Figure $2 \mathbf{C}, \mathbf{F}$ ), after which there was no further increase in micropartcle size with increased temperature. Vacuoles within the heat-treated kafirin microparticles showed a $>10$-fold increase in size to 5-17 $\mu \mathrm{m}$ (Figure 2E, F), compared to the control (Figure 2D). The 
increase in vacuole size with heat treatment was probably due to greater expansion of air with higher temperature within the microparticles, since the vacuoles are probably footprints of air bubbles (2). The relative proportion of microparticles of size $>20 \mu \mathrm{m}$ increased by up to about $40 \%$ with increase in temperature up to $75^{\circ} \mathrm{C}$ (Figure 2C, F). The oval shape of the larger heat-treated microparticles was probably due to the rate of particle coalescence being inversely proportional to particle size, as proposed by (29), who studied the effect of coalescence energy release on the temporal shape evolution of nanoparticles.

SDS-PAGE under non-reducing conditions, of the wet heat treated kafirin microparticles showed no change in band pattern compared with the control until the severity of the treatment was at $96^{\circ} \mathrm{C}$ (Figure 3A). The band pattern under non-reducing conditions of the control, and treatments at $50^{\circ}$ and $75^{\circ} \mathrm{C}$, all show a similar band pattern that is typical of kafirin as described by El Nour et al. (30). The $96^{\circ} \mathrm{C}$ heat treatment, under non-reducing conditions, however, showed a fainter trimer band and disappearance of an oligomer band (Figure 3A, arrows in Lane 4). This is considered indicative of polymerization of the different kafirin species, which then become too large to enter the separating gel (31). The absence of visible change in band pattern at lower treatment temperatures may be due to the electrophoresis technique not being sufficiently sensitive to show a lower degree of polymerization. Under reducing conditions, all the treatments showed similar band patterns with very low levels of kafirin trimers and oligomers. This indicated that the kafirin polymers formed by heat treatment were the result of disulfide crosslinking, and that the linkages were broken on reduction, as demonstrated by the high level of kafirin monomers, as described by (32). 
Concerning kafirin secondary structure, native kafirin is about $60 \% \alpha$-helical (12), whereas Taylor et al. (2) found the secondary structure of kafirin microparticles between $50-56 \% \alpha$-helical. Taylor et al. (2) attributed the lower proportion of $\alpha$-helical structure in kafirin microparticles to protein aggregation during the microparticle formation. The microparticle secondary structure in this study as analyzed by FTIR (Table 1) showed a $\alpha$-helical component of $48.7 \%$ for the control. A number of factors, including different kafirin batches, method of kafirin extraction and drying affect secondary structure measurements (33). There was a further change in secondary structure with heat treatment from 50 to $75^{\circ} \mathrm{C}$, with a progressive reduction in the percentage of $\alpha$-helical conformation, as determined at the Amide I band $\left(\approx 1650 \mathrm{~cm}^{-1}\right)$. This change in protein secondary structure is related to the kafirin polymerization. It has been suggested that thermal treatment disrupts the hydrogen bonds that stabilize the protein conformation, causing loss of the $\alpha$-helix and $\beta$-sheet structures and creating new $\beta$-sheet arrangements (32). Thus, it appears that the increase in size of the kafirin microparticles with increasing heat treatment was as a result of a further process of assisted-assembly caused by wet heat-induced kafirin disulfide bonded polymerization, as described by Emmambux et al. (32).

The wet heat treatment resulted in an up to $39 \%$ reduction in the IVPD of the kafirin microparticles (Table 1). The reduction in IVPD with wet heat treatment is a characteristic of kafirin proteins (34). Reduction in kafirin IVPD has been associated with disulfide bonded polymerization and a reduction in proportion $\alpha$-helical conformation at the amide I band (32), as also observed in this study.

AFM indicated that the microparticles had a surface characterized by a rough morphology (marked X) (Figure 4A-C). Figure 4A illustrates the surface of a single control microparticle. More detailed 
study showed that the surface was composed of nanosized protuberances. Figure 4B shows the surface of a single heat treated microparticle. In this case, more detailed study showed that nanosized protuberances of irregular shape and size (from $50 \mathrm{~nm}$ to $300 \mathrm{~nm}$ ) were responsible for the final surface topography. As viewed by SEM (Figure 2 A-C) the morphology is most likely due to non-linear random aggregation of the structural units (polypeptides). Similar nanostructure aggregation has been reported with zein film drop deposited onto silica (35) and zein nanoparticles precipitated from aqueous ethanol (36). Formation of non-linear aggregate microstructures with heat treatment is a generic property of polypeptides (37).

The variation in size of nanosized protuberances viewed by AFM is probably dependent on how homogenous the particles were in the sites viewed. The aggregated nanosized protuberances viewed from rough areas (R, Figure $\mathbf{4 A - C}$ ) had larger diameter compared to those from flat areas (F, Figure 4A-C). This was probably as result of the broadening phenomenon, where the side of the AFM probe is involved in imaging (38). The broadening effect is due to tip-sample convolution, which results when radius of curvature of the tip is similar to, or greater than, the size of the feature that is imaged (38).

Glutaraldehyde treatment following microparticle preparation. As with increasing severity of wet heat treatment, the average microparticle size increased with severity of the glutaraldehyde treatment, from 1-5 $\mu \mathrm{m}$ the control (Figure 2A, D) to $>20 \mu \mathrm{m}$ with $30 \%$ glutaraldehyde (Figure 2I, L). The relative proportion of microparticles of size $>20 \mu \mathrm{m}$ was also increased, up to about $45 \%$ with 30\% glutaraldehyde (Figure 2I, L). Glutaraldehyde treatment also resulted in particles that were of more elongated oval shape than heat treated microparticles. Unlike heat treatment, the size of the vacuoles in the microparticles did not change with glutaraldehyde treatment (Figure 2J-L). 
This is presumably because there was no heating involved with the glutaraldehyde treatments there was no possibility of expansion of entrapped air.

With SDS-PAGE under non-reducing conditions, increasing severity of glutaraldehyde treatment of the microparticles resulted in a progressive reduction of monomer (16-27 kDa), dimer (44-53 kDa), trimer $(78-93 \mathrm{kDa})$ and oligomer $(>117 \mathrm{kDa})$ (Figure 3B). This is indicative of an increase in kafirin cross-linking with increase in glutaraldehyde concentration, resulting in a reduction in the proportion of kafirin that could enter into the separating gel. Under reducing conditions, the intensity of the kafirin monomer bands was much higher in the control than with the glutaraldehyde treatments. This indicates that the glutaraldehyde cross-linked the kafirin in such a way that breaking of the intermolecular disulfide bonds did not depolymerize it. Thus, the major cause of kafirin cross-linking with glutaraldehyde was not disulfide bonding.

Despite the cross-linking by glutaraldehyde treatment, FTIR indicated that it caused only a small reduction in the relative proportion of $\alpha$-helical conformation (7-9\%) (Table 1). Although crosslinking protein with glutaraldehyde at neutral $\mathrm{pH}$ involves the free amino groups of the proteins and carbonyl groups of the aldehyde, (39), at acid $\mathrm{pH}$ when the amino groups of lysine and proline are protonated, it has been suggested that the reaction may also involve the $-\mathrm{OH}$ groups of hydroxyproline and hydroxylysine leading to the formation of hemiacetals (40). This may help explain the observed small change in protein secondary structure, along with the fact that $\mathrm{N}-\mathrm{H}$ bending contributes only less than $20 \%$ to Amide I band (41). The present findings are consistent with a report on soy protein hydrogels, which showed little alteration in protein secondary structure with glutaraldehyde treatment, despite a large effect on gel physical appearance and functional properties (42). 
Also, despite the glutaraldehyde treatment causing kafirin cross-linking, it did not significantly affect kafirin microparticle IVPD (Table 1). This is consistent with the lack of change in protein secondary structure and is probably a result of an increase in protein free volume caused by the glutaraldehyde cross-linking. It has been suggested that polymeric forms of glutaraldehyde are involved in the cross-linking with protein (43). These glutaraldehyde polymers may create long methylene bridges between peptides (40). Hence, the protein polymer chains may be kept far apart in glutaraldehyde-protein complex, thereby allowing easier accessibility of pepsin enzyme to hydrolyze internal peptide bonds.

AFM also indicated a different cross-linking mechanism for the glutaraldehyde treatment. With the glutaraldehyde treatment, the nanostructures were spindle-shaped with a unidirectional orientation, in contrast to the random irregular shapes seen with heat treatment (Figure 4C). The spindle shaped, aggregated nanostructures were $\approx 100-350 \mathrm{~nm}$ long and $\approx 20-100 \mathrm{~nm}$ wide (Figure 4Ci). The image in Figure 4Cii is probably a cross-section through the spindles represented by circular shapes. No reference to similar shaped protein particle structures visualized by AFM could be found in the literature. The spindle-shape formed with glutaraldehyde treatment suggests a linear polymerization of the kafirin polypeptides during their reaction with glutaraldehyde. This is in agreement with Migneault et al. (43) who suggested that glutaraldehyde-protein reaction results in a cross-linked structure consisting of a linear aldol-condensed oligomer of glutaraldehyde linked to Schiff base (imine) from the protein.

In order to increase the functionality as a hard tissue scaffold, the ability to bind bioactive compounds would be advantageous. BMP-2 was chosen as a model bioactive for further work. 
BMP-2 binding with kafirin microparticles. The rate of BMP-2 binding with all samples was most rapid during the first 30 min of binding, after which the rate of binding slowed down very considerably (Figure 6). BMP-2 binding capacity was in the range of 86-168 ng BMP-2/g kafirin microparticles and 100-140 ng BMP-2/g collagen, depending on the treatment and incubation time. Literature values of BMP-2 binding are highly variable, being both higher and lower than found in this study. For example, recombinant human BMP-2 (rhBMP-2) binding to collagen sponge was negligible at $\mathrm{pH} 3.0$ and 4.0, but levels up to $0.1-0.2 \mathrm{mg}$ rhBMP-2 per mg collagen were recorded at $\mathrm{pH} 5.2$ and $\mathrm{pH} 6.5$ (44). Further, binding capacity values of only 6-7 $\mu \mathrm{g}$ rhBMP-2 per mg PLGA microparticles have been reported (45). Apart from $\mathrm{pH}$, many other factors have been shown to influence BMP-2 binding capacity, some of which may be responsible for the differences between BMP-2 binding capacity in this work and literature values. These include: concentration of BMP-2 used in the binding process, (46) the binding protein $\mathrm{pI}$, (47) ionic strength of the reaction medium (48) and the method of loading of the BMP-2 to the carrier material (45).

However, in this study it was found that irrespective of treatment, after 1440 min of binding the BMP-2 binding capacity of kafirin microparticles was somewhat higher than that of collagen. The BMP-2 binding capacity of the control, heat-treated and glutaraldehyde-treated kafirin microparticles were up to $7 \%, 18 \%$ and $22 \%$ higher than collagen respectively. When compared to the kafirin control, the BMP-2 binding capacity was $10 \%$ higher for the heat treatment and $14 \%$ for the glutaraldehyde treatment. The slightly higher binding capacity of the kafirin mcroparticles to BMP-2 than collagen may be due to the larger surface area of the vacuolated kafirin microparticles. The interaction between BMP-2 and collagen involves electrostatic attraction (47). So in addition, there may have been a stronger interaction between kafirin, pI 6 (49) and BMP-2, pI 9 (48) than 
collagen with a higher pI of 7.8 (50). As kafirin is a relatively hydrophobic protein (12), this may enhance its ability to bind BMP. BMP-2 has been shown to have a strong affinity for hydrophobic surfaces and is believed to be hydrophobic due to hydrophobic pockets formed by BMP-2 monomer residues (51).

The slightly higher BMP binding capacity of treated kafirin microparticles when compared to control kafirin microparticles was probably due to the larger particle size and oval shape of the treated microparticles. Ruhé et al. (45) working with poly(DL-lactic-co-glycolic acid) (PLGA) microparticles reported an increase in BMP-2 entrapment efficiency with increase in particle size.

Kafirin microparticle further assisted-assembly. This study shows that modification of kafirin microparticles with wet heat or glutaraldehyde treatment result in two structures that while similar in size and external morphology, are formed by significantly different mechanisms. It is also apparent that in both cases that the larger kafirin microparticle structures had undergone some form of further assisted-assembly during the treatments and were not just formed as a result of 'gluing' the original microparticles together. The 'gluing together' of microparticles was observed when sorghum condensed tannins were encapsulated using kafirin microparticles (4). Further, with both the heat and glutaraldehyde treatments, there was kafirin cross-linking and although the treatments only resulted in a small changes in secondary structure from $\alpha$ - helical to $\beta$-sheet as shown by FTIR, there was a considerable proportion of $\beta$-sheet present, $59.4 \%$ and $54.9 \%$ for the most rigorous heat treatment and glutaraldehyde treatment, respectively (Table 1). A large proportion of $\beta$-sheet presence is considered indicative of protein aggregation (52) and the universal energetic minimum for aggregated protein (reviewed by 53). Zein can form into aggregates as globules (54), 
fibrils (55), and spherical micro- and nano-particles (11) depending on the conditions of formation, all of which have a large proportion of $\beta$-sheet structure.

Wang and Padua (11) working with zein in 70\% ethanol showed at low mass fraction zein formed spheres. With increasing zein concentration, they noted various different geometries formed by connecting, melting or deformation of spheres. They concluded that spheres were the basis of all other microforms. In this study, the heat-treated microparticles appeared to be formed by coalescence of spherical nanoparticles, in agreement with Wang and Padua (11). Further work by Wang and Padua (10) shows that at a nanoscale, zein formed stripes, rings and discs with a periodicity characteristic of $\beta$-sheet. They indicated that these $\beta$-sheets self-assembled into stripes, which curled into rings and that the rings stacked into spheres. However, in this study AFM indicated the glutaraldehyde-treated microparticles were formed from spindle shaped nanoparticles with little change in secondary structure but with a large proportion of $\beta$-sheet structure present. It appears unlikely that these structures formed from spheres. Further work is needed in order to understand the kafirin assisted-assembly process at a molecular level, under the different conditions used in this study so that the assisted-assembly process can be further manipulated to enable the formation of different structures.

Considering BMP-2 binding to the treated microparticles, both treatments appear to enhance binding. It is probable that this enhancement was due mainly to the increased size and change in shape of the treated microparticles. Thus, these kafirin microparticles have potential as a natural, non-animal protein bio-active scaffolds. More work is needed to determine the release profile of the BMPs and to determine the safety of the material and indeed whether bone morphogenesis can be stimulated with BMP-2 bound to such microparticles in an animal model. 


\author{
ABBREVIATIONS USED \\ BMP - Bone morphogenetic protein \\ SDS - PAGE- Sodium dodecyl sulfate polyacrylamide gel electrophoresis \\ SEM - Scanning Electron Microscopy TEM - Transmission Electron Microscopy \\ AFM - Atomic force microscopy \\ IVPD - In vitro protein digestibility \\ FTIR - Fourier transform infrared spectroscopy \\ ELISA - Enzyme-linked immunosorbent assay
}

\title{
ACKNOWLEDGEMENT
}

The authors thank A. Hall, C. van der Merwe and A. Buys for their assistance with the microscopy.

\section{LITERATURE CITED}

1. Muthuselvi, L.; Dhathathreyan, A. Simple coacervates of zein to encapsulategitoxin. Colloid Surface B 2006, 51, 39-43.

2. Taylor, J.; Taylor, J.R.N.; Belton, P.S. and Minnaar, A. Formation of kafirin microparticles by phase separation from an organic acid and their characterization. J. Cer. Sci. 2009, 50, 90105.

3. Patel, A.; Hu, Y., Tiwari, J.K.; Velikov, K.P. Soft Matter 2010, 6, 6192-6199.

4. Taylor, J.; Taylor, J.R.N.; Belton, P.S.; Minnaar, A. Kafirin Microparticle Encapsulation of Catechin and Sorghum Condensed Tannins. J. Agric. Food Chem. 2009, 57, 7523-7528. 
5. Xiao, D.; Davidson, P.M.; Zhong, Q. Spray dried zein capsules with coencapsulated nisin and thymol as antimicrobial delivery system for enhanced antilisterial properties. J. Agric. Food Chem. 2011, 59, 7393-7404.

6. Parris, N.; Cooke, P.H.; Hicks, K.B. Encapsulation of essential oils in zein nanospherical particles. J. Agric. Food Chem. 2005, 53, 4788-4792.

7. Gong, S.; Wang, H.; Sun, Q.; Xue, S.T.; Wang, J.Y. Mechanical properties and in vitro biocompatibility of porous zein scaffolds. Biomaterials 2006, 27, 3793-3799.

8. Wang, H.J.; Lin, Z.X.; Liu, X.M.; Sheng, S.H.; Wang, J.Y. Heparin-loaded zein microsphere film and hemocompatibility. J. Control Release 2005, 105, 120-131.

9. Reddy, N.; Yang, Y. Potential of plant proteins for medical applications. Trends Biotechnol. 2011, 29, 490-498.

10. Wang, Y.; Padua, G.W. Nanoscale characterisation of zein self-assembly. Langmuir 2012, 28, 2429-2435.

11. Wang, Y.; Padua, G.W. Formation of zein microphases in ethanol-water. Langmuir 2010, 26, 12897-12901.

12. Belton, P.S.; Delgadillo, I.; Halford, N.G.; Shewry, P.R. Kafirin structure and functionality. J. Cer. Sci. 2006, 44, 272-286.

13. Hou, Q.; De Bank, P.A.; Shakeseff, K.M. Injectable scaffolds for tissue regeneration. J. Mat. Chem. 2004, 14, 1915-1935.

14. Weiss, P.; Layrolle, P.; Clergeau, L.P.; Enckel, B.; Pilet, P.; Amouriq, Y.; Daculsi, G.; Giumelli, B. The safety and efficacy of an injectable bone substitute in dental sockets demonstrated in a human clinical trial. Biomaterials 2007, 28, 3295-3305. 
15. Zhang, W.; Zhong, Q. Microemulsions as nanoreactors to produce whey protein nanoparticles with enhanced heat stability by sequential enzymic cross-linking and thermal pretreatments. J. Agric. Food Chem. 2009, 57, 9181-9189.

16. Kim, S.; Kang, Y.; Krueger, C.H.; Sen, M.; Holcomb, J.B.; Chen, D. Wenke, J.C.; Yang, Y. Sequential delivery of BMP-2 and IFG-1 using a chitosan gel with gelatin microspheres enhances early osteoblastic differentiation. Acta Biomater. 2012, 8, 1768-1777.

17. Anyango, J.O.; Taylor, J.; Taylor, J.R.N. Improvement in water stability and other related functional properties of thin cast kafirin films. J. Agric. Food Chem. 2011, 59, 12674-12682.

18. Byaruhanga, Y.B.; Erasmus, C.; Emmambux, M.N.; Belton, P.S.; Wellner, N.; Ng, K.G.; Taylor, J.R.N. Alteration of kafirin film structure by heating with microwave energy and tannin complexation. J. Agric. Food Chem. 2006, 54, 4198-4207.

19. Soliman, E.A.; Eldin, M.S.M.; Furuta, M. Biodegradable zein-based films:Influence of $\gamma$ irradiation on structural and functional properties. J. Agric. Food Chem. 2009, 57, 25292535.

20. Yao, C.; Li, X.; Song, T. Electrospinning and cross-linking of zein nanofiber mats. J. Appl. Polymer Sci. 2006, 103, 380-385.

21. Xu, W.; Karst, D.; Yang, W.; Yang, Y. Novel zein-based electrospun fibers with the water stability and strength for various applications. Polym. Int. 2008, 57, 1110-1117.

22. Jiang, Q.; Reddy, N.; Yang, Y. Cytocompatible cross-linking of electrospun zein fibres for the development of water-stable tissue engineering scaffolds. Acta Biomater. 2010, 6, 40424051.

23. Chen, D.; Zhao, M.; Mundy, G.R. Bone Morphogenetic Proteins. Growth Factors, 2004, 22, 233-241. 
24. Gautschi, O.P.; Frey, S.P.; Zellweger, R. Bone morphogenetic proteins in clinical applications. ANZ J. Surg. 2007, 77, 626-631.

25. Bessa, P.C.; Balmayor, E.R.; Hartinger, J.; Zanoni, G.; Dopler, D.; Meinl, A.; Banerjee, A.; Casal, M.; Redl, H.; Reis, R.L.; van Griensven, M.. Silk fibroin microparticles as carriers for delivery of human recombinant bone morphogenetic protein-2 in vitro and in vivo bioactivity. Tissue Eng. Part C. 2010, 16, 937-945.

26. AACC. Approved Methods of the AACC $10^{\text {th }}$ ed.; American Association of Cereal Chemists: St Paul, MN. 2000.

27. Taylor, J.; Taylor, J.R.N. Alleviation of the adverse effects of cooking on sorghum protein digestibility through fermentation in traditional African porridges. Int. J. Food Sci. Tech. 2002, 37, 129-137.

28. Hamaker, B.R.; Kirleis, A.W.; Mertz, E.T.; Axtell, J.D. Effect of cooking on the protein profiles and in vitro digestibility of sorghum and maize. J. Agric. Food Chem. 1986, 34, 647649.

29. Lehtinen, K.E.J.; Zachariah, M.R. Effect of coalescence energy release on the temporal shape evolution of nanoparticles. Phys. Rev. B 2001, 63, 205402-1-205402-7.

30. E1 Nour, N.A.; Peruffo, A.D.B.; Curioni, A. Characterisation of sorghum kafirins in relations to their cross-linking behavior. J. Cer. Sci. 1998, 28, 197-207.

31. Ezeogu, L.I.; Duodu, K.G.; Taylor, J.R.N. Effects of endosperm texture and cooking on the in vitro starch digestibility of sorghum and maize flours. J. Cer. Sci. 2005, 42, 33-44.

32. Emmambux, N.M.; Taylor, J.R.N. Properties of heat-treated sorghum and maize meal and their prolamin proteins. J. Agric. Food Chem. 2009, 57, 1045-1050. 
33. Gao, C.; Taylor, J.; Wellner, N.; Byaruhanga, Y.B.; Parker, M.L.; Mills, E.M.C.; Belton, P.S. Effect of preparation conditions on protein secondary structure and biofilm formation of kafirin. J. Agric. Food Chem. 2005, 53, 306-312.

34. Duodu, K.G.; Taylor, J.R.N.; Belton, P.S.; Hamaker, B.R. Factors affecting sorghum protein digestibility. J. Cer. Sci. 2003, 38, 117-131.

35. Panchapakesan, C.; Sozer, N.; Dogan, H.; Huang, Q.; Kokini, J.L. Effect of different fractions of xeon on the mechanical and phase properties of zein films at nano-scale. J. Cer. Sci. 2012, 55, 174-182.

36. Xu, H.; Jiang, Q.; Reddy, N.; Yang, Y. Hollow nanoparticles from zein for potential medical applications. J. Mater. Chem. 2011, 21, 18227-18235.

37. Krebs, M.R.H.; Devlin, G.L.; Donald, A.M. Protein particulates: Another generic form of protein aggregation? Biophys. J. 2007, 92, 1336-1342.

38. Shakesheff, K.M.; Davies, M.C.; Jackson, D.E.; Roberts, C.J.; Tendler, S.J.B.; Brown, V.A.; Watson, R.C.; Barrett, D.A.; Shaw, P.N. Imaging the surface of silica microparticles with the atomic force microscope: a novel sample preparation method. Surf. Sci. Lett. 1994, 304, L393-L399

39. Rayment, I. Reductive alkylation of lysine residues to alter crystallization properties of properties. Methods Enzymol. 1997, 276, 171-179.

40. Farris, S.; Song, J.; Huang, Q. Alternative reaction mechanism for the cross-linking of gelatin with glutaraldehyde. J. Agric. Food Chem. 2010, 58, 998-1003.

41. Pelton, J.T.; McLean, L.R. Spectroscopic methods for analysis of protein secondary structure. Anal. Biochem. 2000, 277, 167-176. 
42. Caillard, R.; Remondetto, G.E.; Subirade, M. Physicochemical properties and microstructure of soy protein hydrogels co-induced by Maillard type cross-linking and salts. Food Res. Int. 2009, 42, 98-106.

43. Migneault, I.; Dartiguenave, C.; Bertrand, M.J.; Waldron, K.C. Glutaraldehyde: behavior in aqueous solution, reaction with proteins, and application to enzyme crosslinking.

Biotechniques 2004, 37, 790-802.

44. Friess, W.; Uludag, H.; Foskett, S.; Biron, R.; Sargeant, C. Characterization of absorbable collagen sponges as rhBMP-2 carriers. Int. J. Pharm. 1999, 187, 91-99.

45. Ruhé, P.Q.; Boerman, O.C.; Russel, F.G.M.; Spauwen, P.H.M.; Mikos, A.G.; Jansen, J.A. Controlled release of rhBMP-2 loaded poly(DL-lactic-co-glycolic acid)/calcium phosphate cement composites in vivo. J. Control Release 2005, 106, 162-171.

46. Schrier, J.A.; DeLuca, P.P. Recombinant human bone morphogenetic protein-2 binding and incorporation in PLGA microsphere delivery systems. Pharm. Dev. Technol. 1999, 4, 611621.

47. Patel, Z.S.; Yamamoto, M.; Ueda, H.; Tabata, Y.; Mikos, A.G. Biodegradable gelatin microparticles as delivery systems for the controlled release of bone morphogenetic protein2. Acta Biomater. 2008, 4, 1126-1138.

48. Geiger, M.; Li, R.H.; Friess, W. Collagen sponges for bone regeneration with rhBMP-2. Adv. Drug Delivery Rev. 2003, 55, 1613-1629.

49. Csonka, F.A.; Murphy, J.C.; Jones, D.B. The iso-electric points of various proteins. J. Am. Chem. Soc. 1926, 48, 763-768.

50. Higberger, J.H. The isoelectric point of collagen. J. Am. Chem. Soc. 1939, 61, 2302-2303. 
51. Nickel, J.; Dreyer, M.K.; Kirsch, T.; Sebald, W. The crystal structure of the BMP-2:BMPRIA complex and the generation of BMP-2 antagonists. J. Bone Joint Surg. 2001, 83-A (Suppl. 1, Part 1), S7-S14.

52. Mizutani, Y.; Matsumura, Y.; Imamura, K.; Nakanishi, K.; Mori, T. Effects of water activity and lipid addition on secondary structure of zein in powder systems. J. Agric. Food Chem. 2003, 51, 229-235.

53. Gorbenko, G.P.; Kinnunen, P.K.J. The role of lipid-protein interactions in amyloid-type protein fibril formation. Chem. Phys. Lipids 2006, 141, 72-82.

54. Guo, Y.; Liu, Z.; An, H.; Li, M.; Hu, J. Nano-structure and properties of maize zein studied by atomic force microscopy. J. Cer. Sci. 2005, 41, 277-281.

55. Erickson, D.P.; Campanella, O.H.; Hamaker, B.R. Functionalizing maize zein in viscoelastic dough systems through fibrous, $\beta$-sheet-rich protein networks: An alternative, physiochemical approach to gluten-free breadmaking. Trends Food Sci. Tech. 2012, 24, 7481.

Joseph Anyango is grateful for the provision of a University of Pretoria Postgraduate Research Support Bursary. 
Table 1. Effects of wet heat and glutaraldehyde treatments on the protein secondary structure of kafirin microparticles determined by FTIR and in vitro protein digestibility (IVPD)

\begin{tabular}{lccc}
\hline Treatment & $\begin{array}{c}\text { Relative proportion of } \\
\text { a-helical conformation at } \\
\text { Amide I band } \\
(\%)\end{array}$ & $\begin{array}{c}\text { IVPD } \\
(\%)\end{array}$ \\
\hline Control & $22^{\circ} \mathrm{C}$ & $48.7 \mathrm{~d}(0.6)$ & $94.4 \mathrm{~d}(0.9)$ \\
Heat & & $45.5 \mathrm{c}(0.3)$ & $90.2 \mathrm{c}(1.8)$ \\
& $50^{\circ} \mathrm{C}$ & $40.9 \mathrm{a}(0.2)$ & $73.5 \mathrm{~b}(0.7)$ \\
& $75^{\circ} \mathrm{C}$ & $40.6 \mathrm{a}(0.5)$ & $57.3 \mathrm{a}(1.5)$
\end{tabular}

Glutaraldehyde

$\begin{array}{lll}10 \% & 44.5 \mathrm{~b}(0.6) & 91.3 \mathrm{~cd}(1.8) \\ 20 \% & 45.1 \mathrm{bc}(0.2) & 91.9 \mathrm{~cd}(2.8) \\ 30 \% & 45.1 \mathrm{bc}(0.8) & 92.5 \mathrm{~cd}(0.3)\end{array}$

Values in a column followed by different letters are significantly different $(\mathrm{p}<0.05)$.

Values in brackets are standard deviations $(\mathrm{n}=3)$. Amide I band $\approx 1650-1620 \mathrm{~cm}^{-1}$ 


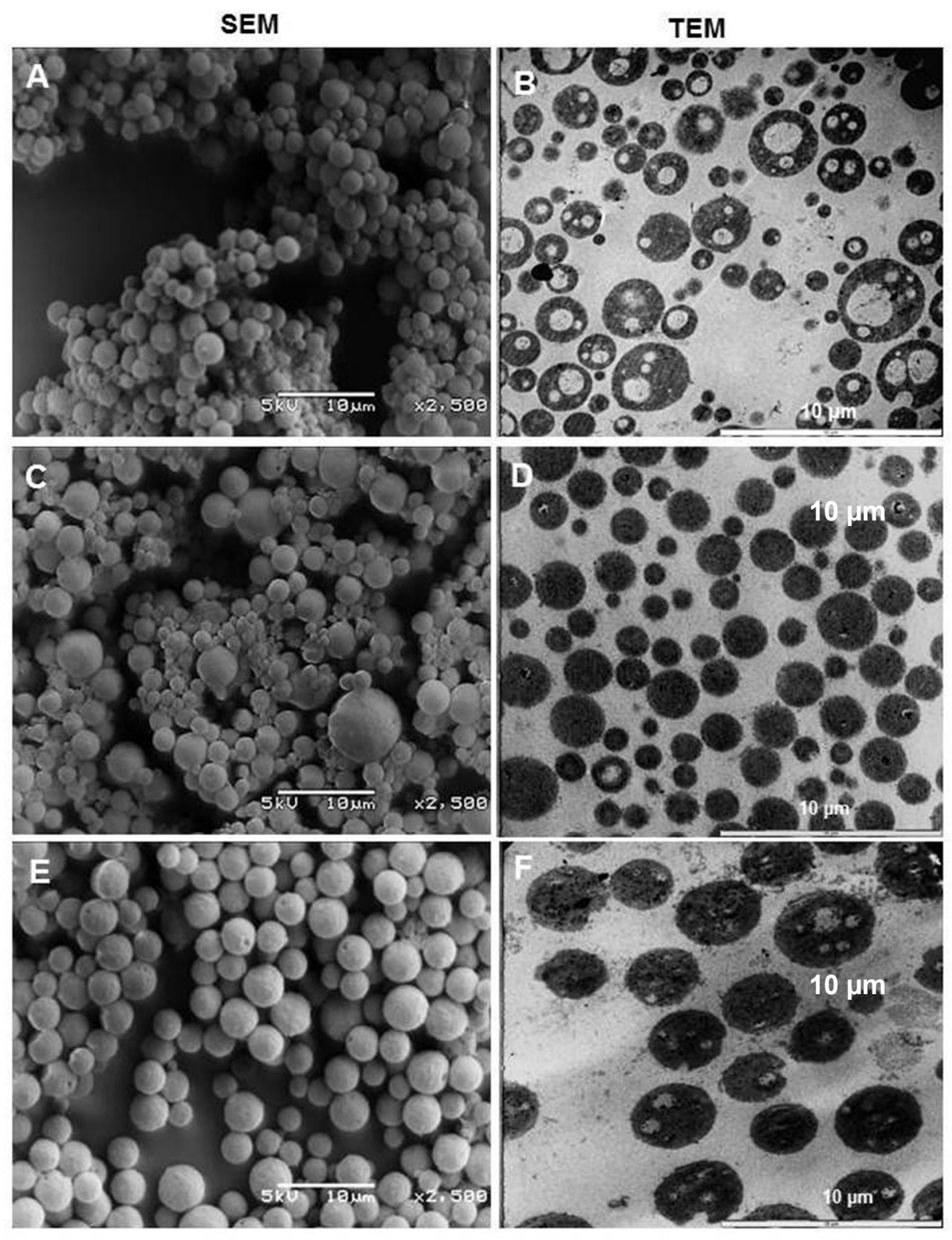

Figure 1. Electron microscopy of kafirin microparticles treated during preparation. (A, C, and E) SEM and (B, D, and F) TEM. (A and B) Control, (C and D) heat treatment, and (E and F) glutaraldehyde treatment. 

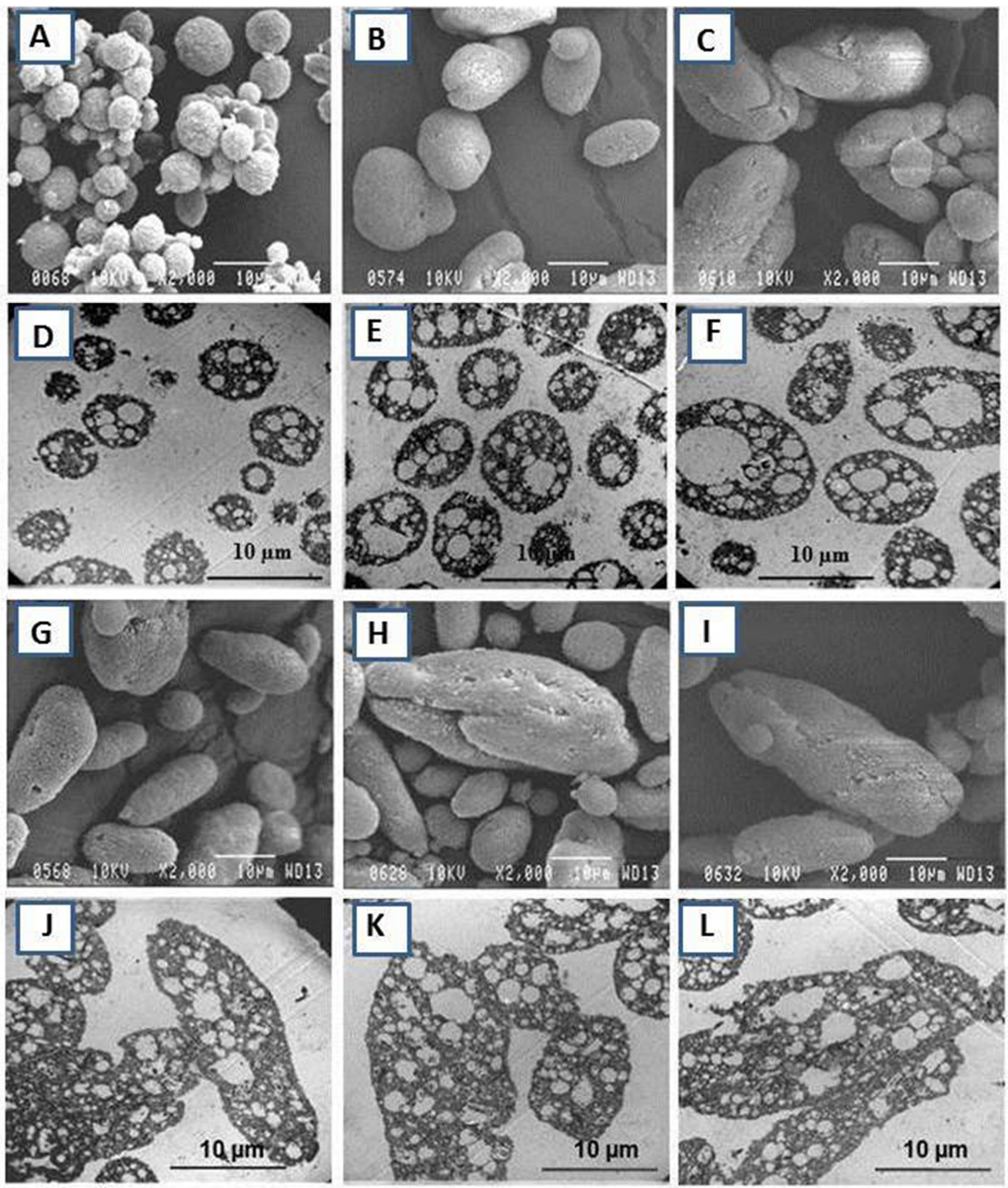

Figure 2. Electron microscopy of kafirin microparticles treated after preparation. $(\mathrm{A}-\mathrm{C}$ and $\mathrm{G}-\mathrm{I}) \mathrm{SEM}$ and (D-F and J-L) TEM. (A and D) Control, heated; (B and E) $50{ }^{\circ} \mathrm{C}$; and (C and F) $75^{\circ} \mathrm{C}$. Glutaraldehyde: (G and J) 10\%, (H and K) 20\%, and (I and L) $30 \%$. 

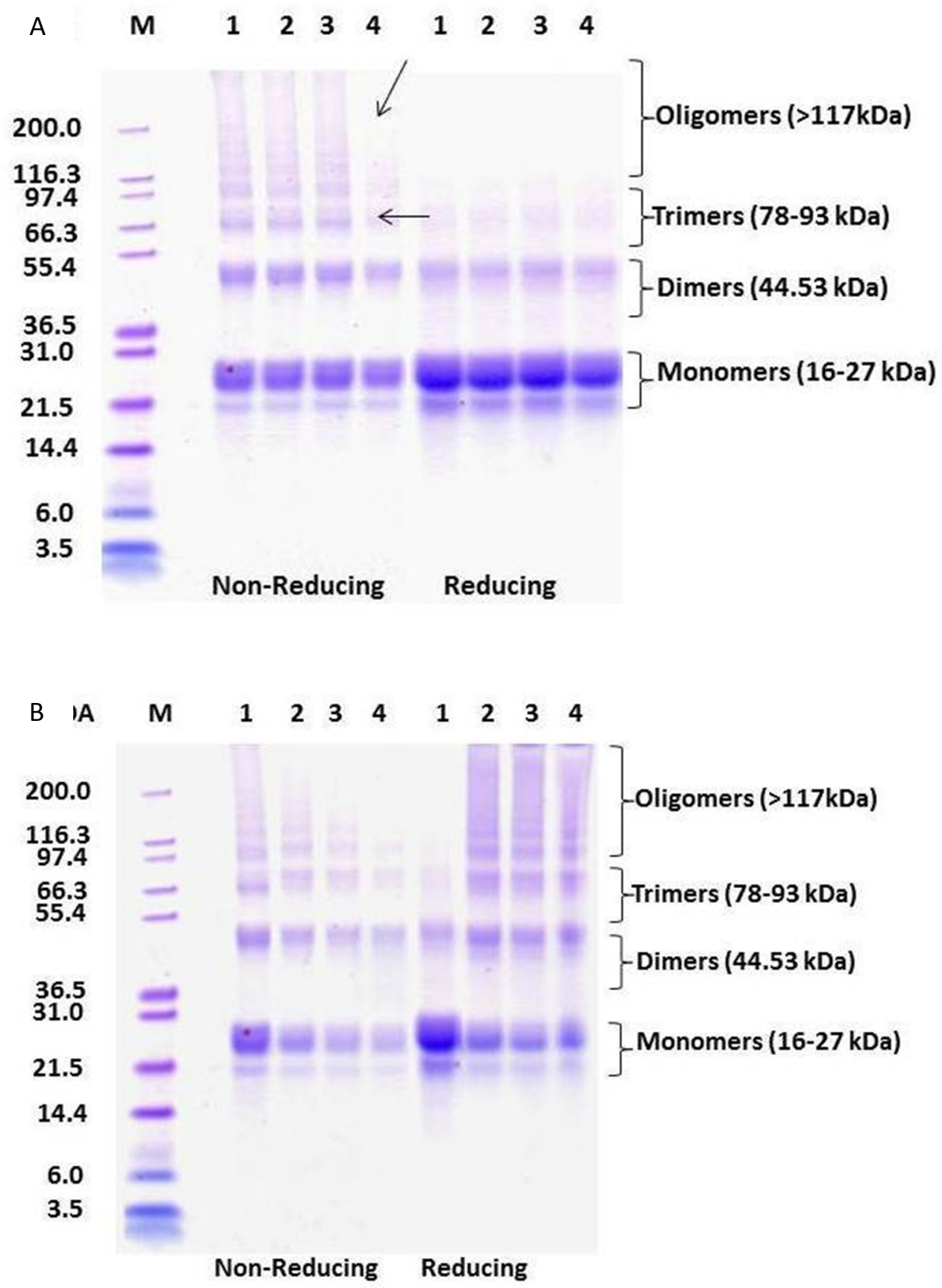

Figure 3. SDS-PAGE of treated kafirin microparticles. Protein loading, $10 \mu \mathrm{g}$. (A) Wet heat treatment. Lanes: M, molecular markers; 1 , control $\left(22^{\circ} \mathrm{C}\right) ; 2,50{ }^{\circ} \mathrm{C} ; 3,75{ }^{\circ} \mathrm{C}$; and $4,96{ }^{\circ} \mathrm{C}$. The arrows in lane 4 under nonreducing conditions show fading and disappearance of bands. (B) Glutaraldehyde treatment. Lanes: M, molecular markers; 1, control; 2, 10\%; 3, 20\%; and $4,30 \%$. 
A
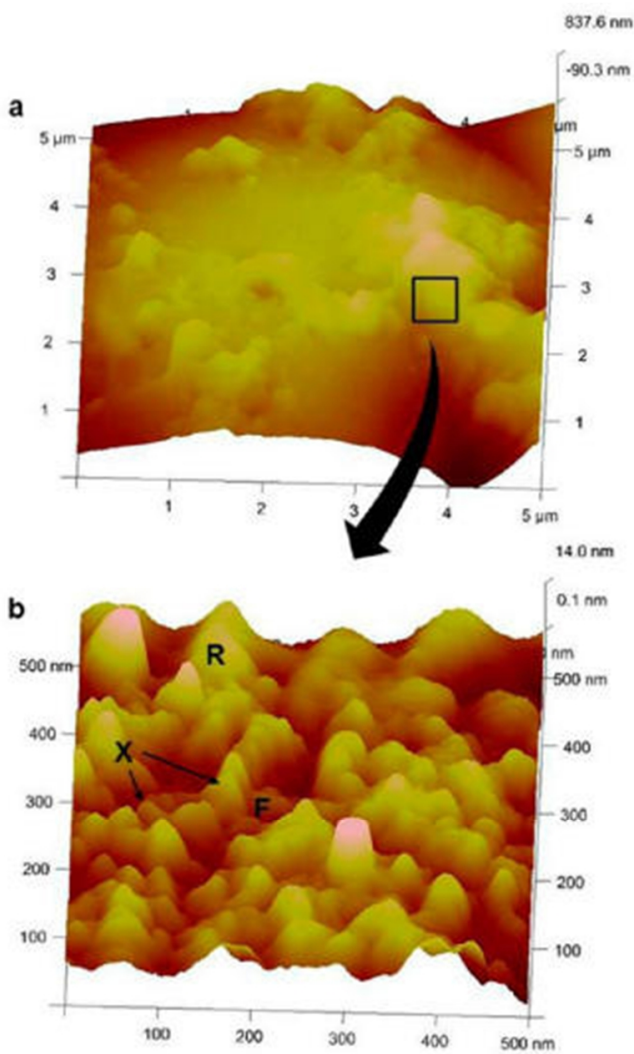

B
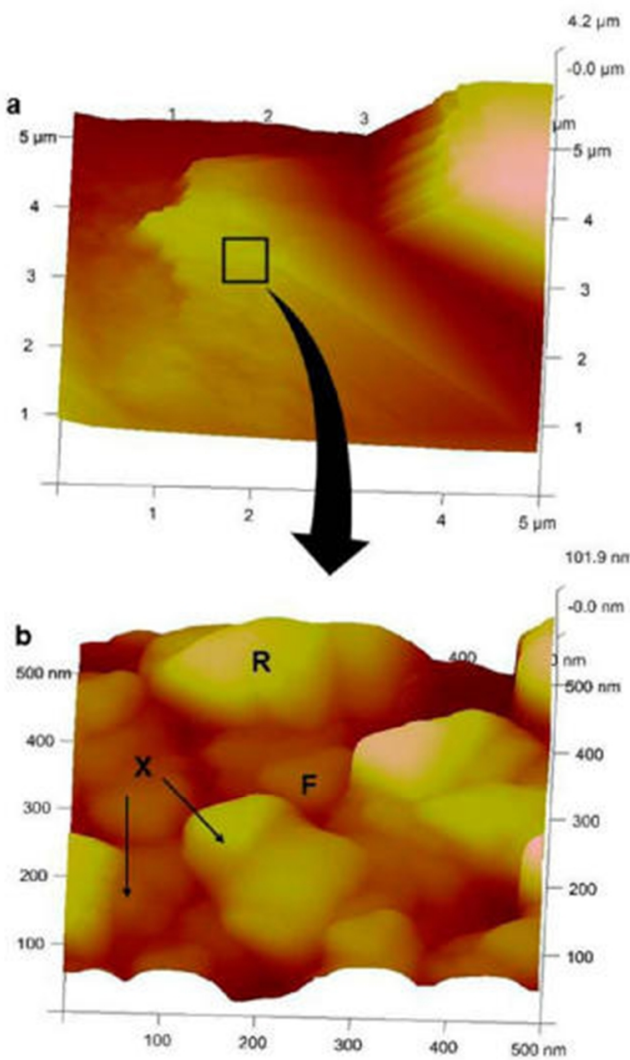

C

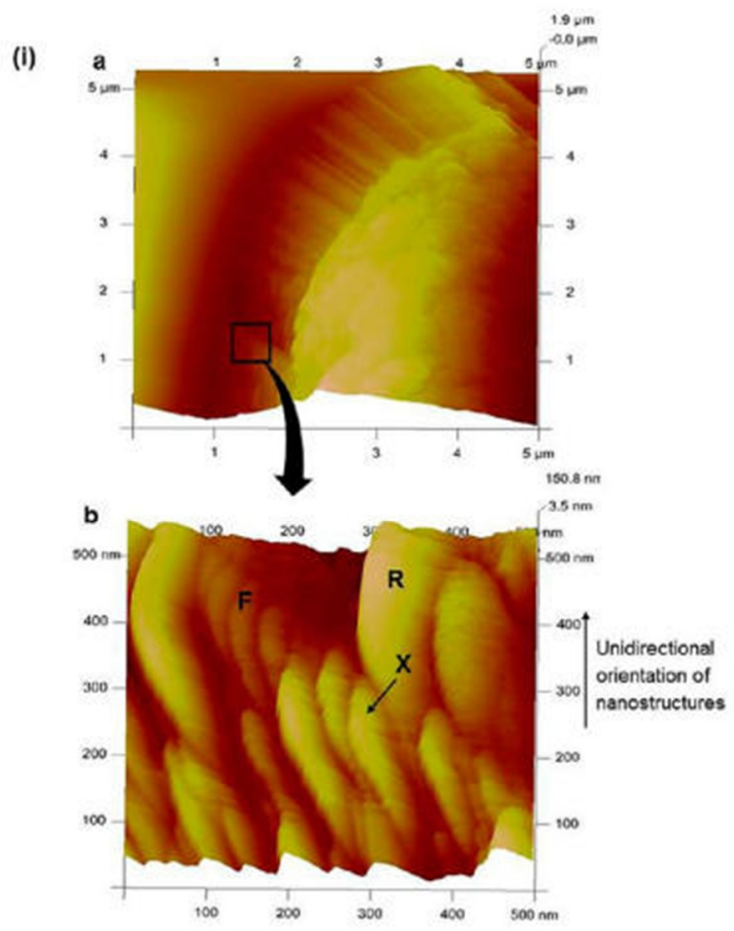

Figure 4. AFM topographs of treated kafirin microparticles at two different levels of magnification. (a) Lower magnification and (b) higher magnification. (A) Control $\left(22{ }^{\circ} \mathrm{C}\right)$, (B) wet heat treatment $\left(96^{\circ} \mathrm{C}\right)$, and (C) glutaraldehdye treatment (30\%). (i) Top view and (ii) view from end of nanostructures: $\mathrm{F}$, flat area; $\mathrm{R}$, rough area; $\mathrm{X}$, and aggregated nanostructures. 


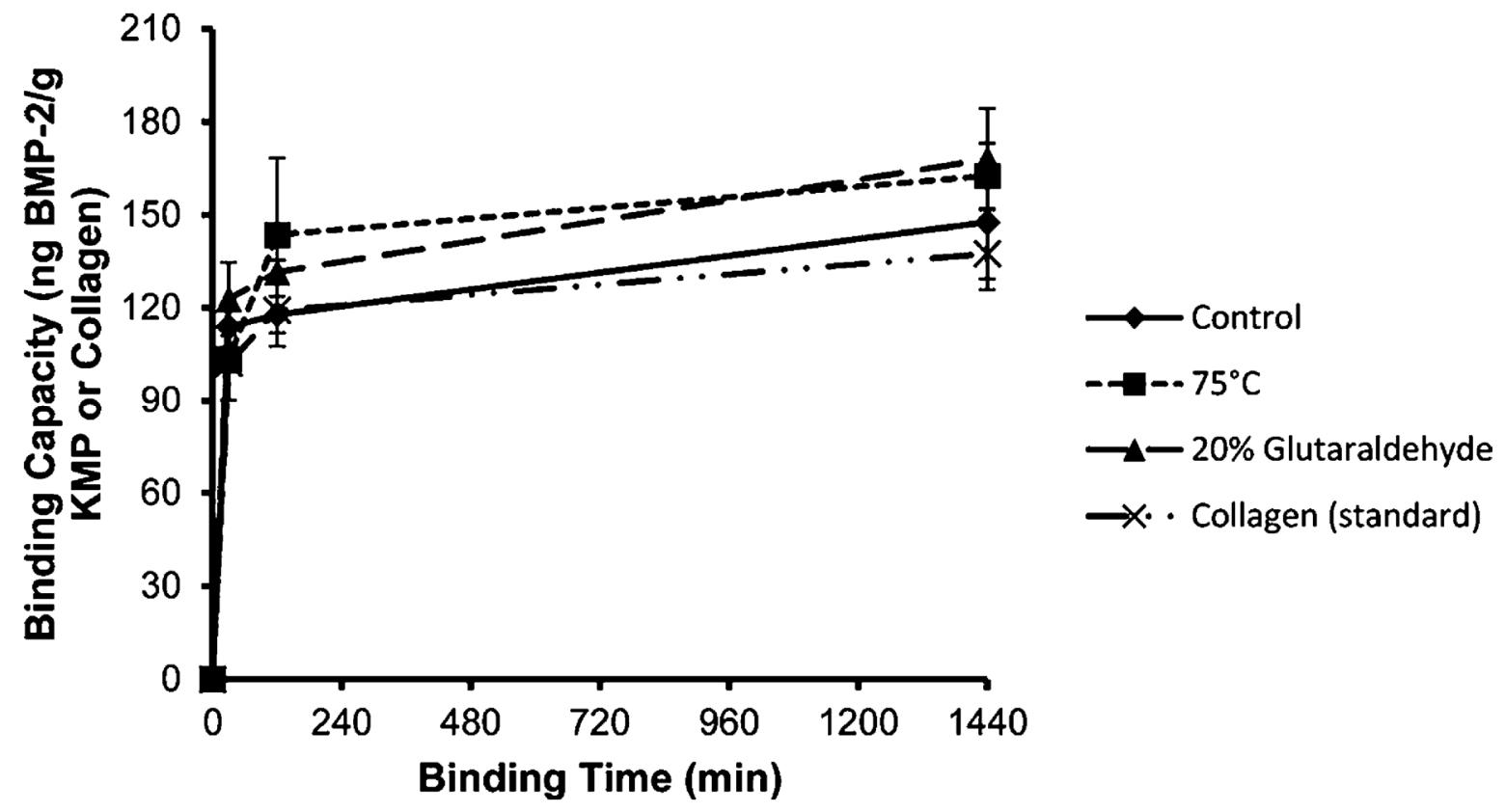

Figure 5. Relative BMP-2 binding capacity of wet heat and glutaraldehyde-treated kafirin microparticles (KMP) as compared with collagen standard over a $24 \mathrm{~h}$ reaction period, determined by ELISA. Curves are plotted using mean relative BMP-2 binding capacity at the set time intervals. Error bars are standard deviations $(n=2)$. 